\title{
Archives
}

\section{La subjectivité dans la numérisation : les perspectives des professionnels}

\section{Joshua Chalifour et Eun G. Park}

Volume 47, numéro 1, 2017

URI : https://id.erudit.org/iderudit/1041825ar

DOI : https://doi.org/10.7202/1041825ar

Aller au sommaire du numéro

Éditeur(s)

Association des archivistes du Québec (AAQ)

ISSN

0044-9423 (imprimé)

2369-9256 (numérique)

Découvrir la revue

Citer cet article

Chalifour, J. \& Park, E. G. (2017). La subjectivité dans la numérisation : les perspectives des professionnels. Archives, 47(1), 31-58.

https://doi.org/10.7202/1041825ar
Résumé de l'article

L'influence de la subjectivité sur les processus de sélection en matière de numérisation n'a pas encore été étudiée en profondeur. La présente étude traite des facteurs subjectifs qui sous-tendent les choix des professionnels engagés dans des projets de numérisation ; entreprend une réflexion sur la manière dont ils prennent des décisions de sélection et examine dans quelle mesure leurs points de vue influencent les sélections. Des entrevues menées auprès de cinq professionnels des bibliothèques ou centres d'archives révèlent six facteurs subjectifs récurrents. La documentation de l'influence de ces facteurs apporte davantage de clarté, offrant une meilleure compréhension des objets numériques. 


\section{ÉTUDE}

\section{La subjectivité dans la numérisation: les perspectives des professionnels}

\section{JOSHUA CHALIFOUR}

Bibliothécaire, Bibliothèques de I’Université Concordia

\section{EUN G. PARK}

Professeur agrégé ; École d'études de l'information, Université McGill

Traduction par Bruce Henry

Révision par Julie Le Bouthillier et Susanne Julien

\section{INTRODUCTION}

Les archivistes et les bibliothécaires qui entreprennent des projets de numérisation ont leurs propres motivations pour créer des objets numériques, mais ces raisons peuvent être subjectives et ne reposent donc pas uniquement sur des facteurs objectifs. Ni l'influence de la subjectivité sur la sélection pour la numérisation, ni la manière de prendre en compte cette influence dans sa réception par l'utilisateur n'ont été explicitement et pleinement explorées. 
La numérisation permet d'accéder aux contenus existants via des substituts numériques. La création de ces substituts résulte généralement d'un processus de sélection des sources analogiques qui est basé sur des facteurs objectifs, clairs et concrets. Dans de nombreux guides de numérisation, les instructions sont imprégnées des perspectives archivistiques traditionnelles de neutralité et d'impartialité. Bien que certains guides indiquent clairement que la subjectivité influence la phase de sélection de la numérisation, ils nous laissent incertains sur la façon de jauger le rôle subjectif joué par les professionnels d'archives et de bibliothéconomie lors de la sélection d'un projet de numérisation.

Le raisonnement des archivistes ou des bibliothécaires qui créent des objets numériques n'est pas évident ou transparent pour les utilisateurs qui accèdent aux objets. Lors de la sélection d'enregistrements, de fonds ou de collections pour la préservation numérique, les professionnels doivent tenir compte de nombreux facteurs qui favorisent l'entretien et l'accès à I'objet numérique résultant. (Association for Library Collections \& Technical Services, 2007) II est important que ces professionnels réfléchissent à la manière dont les futurs utilisateurs recevront intellectuellement ce qui a été sélectionné.

Cette étude a un triple objectif:

- recueillir auprès d'archivistes et de bibliothécaires qui ont participé à des projets de numérisation des informations sur la façon dont ils ont fait des jugements de sélection;

- identifier des thèmes communs dans leurs expériences qui pourraient, en quelque sorte, recevoir un genre de reconnaissance formelle;

- analyser les éléments de la subjectivité afin de compléter les orientations existantes sur les choix que font les archivistes et les bibliothécaires dans les projets de numérisation.

Avec cette étude, nous espérons ainsi identifier l'espace d'innovation dans le processus de numérisation afin de rendre la subjectivité plus transparente.

Cet article aborde les lignes directrices, le profil des responsabilités professionnelles et l'impact de la subjectivité comme ces sujets sont traités dans la littérature courante. II explique la méthodologie et les résultats de l'étude et discute des questions pertinentes. Les résultats de cette étude 
nous suggèrent quelques recommandations sur la subjectivité et sur la recherche approfondie de ce sujet.

\section{REVUE DE LA LITTÉRATURE}

\subsection{Lignes directrices actuelles}

La numérisation est le processus consistant à « transformer l'information analogique (de toute forme et sur quelque support que ce soit) en code numérique » (Conseil canadien des archives, 2002). Dans la numérisation, l'étendue du processus de sélection passe du choix des documents à numériser, à leur évaluation et ensuite à la hiérarchisation des documents choisis. Des projets de conversion numérique gérés de cette façon sont décrits dans le manuel du Centre de conservation des documents du NordEst (NEDCC) des États-Unis (Sitts, 2000). Quelques critères du processus de sélection de la NEDCC sont: I'évaluation de l'entretien continu des documents à la lumière de la mission de l'établissement et à la portée de sa collection; les exigences légales; la valeur en termes d'authenticité, d'effort, d'audience, etc. ; et des préoccupations physiques et techniques.

D'autres guides et documents sur les meilleures pratiques traitent de la même façon le processus de sélection. À titre d'exemple, les lignes directrices du Royaume-Uni sur les archives parlementaires (United Kingdom. Parliamentary Archives, 2008), les lignes directrices sur la conservation numérique élaborées pour la Commission européenne (Fresko et Tombs, 1998) et le guide du Réseau canadien d'information sur le patrimoine concernant la planification et la mise en œuvre de projets de numérisation (Réseau canadien d'information sur le patrimoine, 2000) détaillent les pratiques de sélection pour la numérisation. Les facteurs de sélection de ces guides ont tendance à être objectifs plutôt que subjectifs. C'est-à-dire que les guides ont tendance à se concentrer sur des facteurs liés aux aspects matériaux des objets. Ils identifient les facteurs relatifs aux phénomènes, qui sont indépendants des perspectives personnelles et des attitudes (c'est-à-dire la subjectivité) des professionnels qui font la sélection.

Certains guides traitant du processus de numérisation sont moins explicites quant aux facteurs de sélection, mais abordent la subjectivité. À titre d'exemple, la déclaration de treize principes du Conseil canadien des archives énonce des vérifications concrètes en accord avec les 
politiques institutionnelles et fournit des énoncés généraux pour influencer la conduite des activités des archivistes dans la numérisation (Conseil canadien des archives, 2002). La déclaration ne traite pas explicitement des facteurs subjectifs, mais permet d'élaborer des objectifs de projet qui peuvent être atteints en fonction de la subjectivité du professionnel. La Digitization Guidelines Initiative des organismes fédéraux des États-Unis recommande que les propositions d'inscription soient fondées sur les priorités institutionnelles et sur les intérêts des chercheurs ou du grand public (Still Image Working Group, 2009).

La publication britannique Preparing Collections for Digitization identifie quelques facteurs primordiaux et objectifs de sélection et, une fois qu'ils sont satisfaits, en recommande d'autres, y compris le contenu, la demande et la condition physique. Elle identifie en outre des moyens d'évaluer ces facteurs, comme l'obtention des connaissances du personnel de l'établissement ou encore lancer une invitation au public et aux groupes d'utilisateurs pour proposer des documents. En raison de l'origine potentiellement subjective d'où proviennent les propositions, les auteurs recommandent qu'un groupe d'experts les examine afin d'obtenir autant de points de vue différents que possible (Bülow, Ahmon et Spencer, 2011).

L'évaluation des questions liées aux contenus qui seraient dits sensibles pour les parties prenantes sur le plan éthique ou sur le plan culturel implique une focalisation subjective. Vogt-O'Connor suppose que les sensibilités éthiques et culturelles vont peser sur les jugements des archivistes et des bibliothécaires. En effet, ces professionnels auront une position sur la limite jusqu'où ils peuvent participer activement à la sphère publique (Vogt-O'Connor, 2000).

L'évaluation conduit à des problèmes délicats de subjectivité, mais quelques guides traitent de ces questions. À titre d'exemple, le guide de la National Initiative for a Networked Cultural Heritage des États-Unis (NINCH) reconnaît que des facteurs, tels que la valeur intellectuelle, impliquent des jugements subjectifs. Le guide de la NINCH présente dix questions à poser avant de sélectionner le matériel à numériser. Ces questions abordent le contenu en ce qui concerne la signification intellectuelle, le matériel exemplaire, l'originalité, l'exhaustivité, l'épreuve du temps, etc. Le guide recommande de demander le consensus des parties prenantes parce que les perceptions ou l'évaluation peuvent varier d'une personne à I'autre (un clin d'œil à la subjectivité) (Moss, 2005). Un guide commandé 
par I'UNESCO recommande que chaque projet formule une définition de la valeur intellectuelle conforme à ses objectifs (Mcllwaine et al., 2002). Idéalement, les utilisateurs considéreront que le contenu vaut l'effort et les ressources nécessaires à sa numérisation. La politique de préservation des Archives nationales du Royaume-Uni analyse la valeur en termes de qualités qui comprennent ses informations, son aspect matériel (l'âge, la provenance, sa condition physique), et la culture (politique, esthétique et éthique) (United Kingdom. The National Archives, 2009). Enfin, Lorna Hughes demande de déterminer si le contenu informationnel et la valeur intellectuelle des documents valent la numérisation (Hughes, 2004). Ces approches pour déterminer la valeur impliquent des jugements qui sont de nature subjective.

\subsection{Subjectivité à l'égard des responsabilités professionnelles}

La numérisation implique des responsabilités que les archivistes et les bibliothécaires ont toujours eues, ainsi que de nouvelles spécificités du domaine numérique. Nous avons besoin d'un sens des responsabilités à la fois traditionnelles (à partir des objets analogiques) et nouvelles (pour les objets numériques).

Dans la perspective traditionnelle, Jenkinson s'est opposé aux jugements personnels comme étant préjudiciables à la réputation d'impartialité d'un service d'archives (Jenkinson, 1922). Schwartz et Cook ont observé que «dans la profession, les archivistes se sont perçus comme neutres, objectifs, impartiaux» (Schwartz et Cook, 2002).

Contrairement à ce point de vue sur l'impartialité, Cook soutient que les archivistes imprègnent leur travail de leurs propres valeurs, ce qui exige un examen attentif des processus de construction des archives. II déclare que «les archives consistent en une fiducie publique sacrée de préserver les souvenirs de la société qui doivent être largement partagés. Les archivistes servent la société »(Cook, 2001). Accepter cette caractérisation suggère que les professionnels ont la responsabilité d'analyser comment leurs propres jugements des souvenirs de la société se rapportent au service à la société. Cette responsabilité n'est pas tout à fait comprise dans le cadre traditionnel de l'impartialité.

D'autres études appuient le point de vue selon lequel les professionnels doivent faire valoir leur influence. À titre d'exemple, Roosa discute de 
l'évolution des attitudes à l'égard des programmes de préservation qui comprennent certaines techniques numériques et il soutient que les bibliothécaires de conservation ont de nombreuses nouvelles capacités qu'ils doivent développer afin de traiter les problèmes futurs, y compris des capacités philosophiques. La numérisation de la Bible de Gutenberg de la Bibliothèque du Congrès des États-Unis a ouvert aux étudiants, aux chercheurs et aux autres personnes une nouvelle compréhension de la signification de l'objet et de l'impact de l'innovation de Gutenberg. L'accessibilité de cet objet numérique a renforcé la compréhension de l'impact de l'invention de Gutenberg. La disponibilité de l'objet numérique stimule la conscience et la visibilité des sujets qui y sont liés, et non seulement de l'objet lui-même. Pour Roosa, les professionnels sont tenus d'assumer la responsabilité de considérer l'impact de leurs choix. «Avant tout, les programmes de préservation devraient être des forces motrices qui guident et coordonnent de façon responsable l'art, l'artisanat et la science de la préservation »(Roosa, 2005).

La numérisation implique une charge de travail accrue lors de la sélection pour la conservation. Gertz parle de cette augmentation du travail, en déclarant qu' « utiliser la numérisation pour donner l'accès à des matériaux de valeur à long terme requiert un véritable engagement à préserver les fichiers numériques [...] régulièrement au long terme» (Gertz' 2000). Dans le cadre de ces paramètres, les jugements effectués lors de la sélection ne se limitent pas à ce que les utilisateurs reçoivent, mais lient aussi les archivistes et les bibliothécaires à de nouvelles responsabilités.

\subsection{L'impact de la subjectivité}

La sélection pour la numérisation, influencée par les considérations des archivistes et des bibliothécaires en choisissant ce qui sera finalement présenté, forme le discours autour de la matière. Les documents sélectionnés pour un projet de numérisation peuvent être présentés ultérieurement dans des contextes différents. Ce qui aura une incidence sur la façon dont les utilisateurs ou les chercheurs percevront leur contenu (Hampshire et Johnson, 2009). La sélection peut être axée sur des documents ayant un contenu spécifique à cause d'un besoin de complémenter une collection numérique axée sur des sujets. Ceci est observé dans les projets de numérisation collaborative où chaque collection sera la source d'une partie des matériaux numérisés (Zhang et al., 2008). 
Cela exige que les professionnels jugent la pertinence du contenu et suggèrent qu'ils anticipent des domaines spécifiques de recherche ou d'utilisation qui s'appliqueront à l'objet numérisé (la subjectivité est implicite dans les deux activités). Ainsi, le processus de sélection est influencé par le but de créer quelque chose à des fins qui n'étaient pas inhérentes aux matériaux originaux.

De même que la sélectivité dans le processus d'évaluation des documents $d$ 'archives originaux favorise certaines caractéristiques des documents dans leur ensemble, la sélectivité du processus de numérisation influence le caractère de ce que les utilisateurs recevront sur le plan intellectuel des nouveaux objets numériques. Verne Harris regardait la tendance à penser que les archives reflètent une réalité où l'archiviste est essentiellement neutre. II a contesté cette tendance en se servant d'exemples d'archives sud-africaines par rapport au système d'apartheid. Harris montre que les archivistes créent de la valeur, en utilisant leurs perspectives et leurs interactions pour façonner le dossier archivistique pendant le processus d'évaluation (un type de sélection) (Harris, 2002).

Les documents sélectionnés deviennent accessibles pour la recherche, mais les contraintes de temps et de ressources pour les numériser signifient que la sélection d'une collection ou d'un fonds élimine la possibilité de la sélection d'autres fonds. Ce qui entraîne le risque que ces derniers ne soient disponibles pour une utilisation future (Anderson, 2006). Cette sélection peut également représenter un défi pour la compréhension du contenu en dehors du contexte complet de la collection (Smith, 1999). McElrath montre qu'un véritable acte d'influence sur la sphère publique vient des choix du professionnel. II démontre que lorsqu'un archiviste choisit de numériser (rendre ainsi accessible) un élément de notre histoire, il augmente la conscience des gens sur cette partie de I'histoire (McElrath, 2007).

Cole et Hackett, dans leur article sur la recherche dans l'environnement numérique, jettent un coup d'œil sur les attentes des chercheurs concernant ce qui est intellectuellement recevable et ce qui est effectivement fourni dans un contexte numérique (Cole et Hackett, 2010). En soi, I'objet sélectionné forme ce que les utilisateurs seront en mesure de rechercher et même son contexte. Ce que Sternfeld souligne tout en considérant le rôle créatif et personnel que les professionnels jouent en sélectionnant des dossiers d'événements historiques (Sternfeld, 2011). En raison de la recherche et du comportement de recherche des utilisateurs, ainsi que des 
attentes quant à la disponibilité des documents numérisés, les archivistes et les bibliothécaires doivent tenir compte de la perception de l'objet numérique qui résulte de leur décision.

La littérature professionnelle identifie les domaines dans lesquels la subjectivité des professionnels de l'information influence la sélection des matériaux à numériser. Ce qui est sélectionné pour la numérisation a une incidence sur la perception que les utilisateurs ont du contenu numérique lorsqu'ils y accèdent. Les lignes directrices existantes offrent beaucoup de détails lorsqu'on aborde des facteurs de sélection objectifs. Mais quant à la subjectivité, la littérature ne fait qu'isoler des questions sur notre façon d'aborder le processus de sélection et ne résout pas notre réflexion sur son impact.

\section{2. ÉTUDE SUR LA SUBJECTIVITÉ}

\subsection{La méthodologie}

Cette étude a exploré la subjectivité au cours du processus de sélection pour la numérisation. Elle a recueilli des informations auprès d'archivistes et de bibliothécaires qui travaillent à la numérisation, a cherché à reconnaître des thèmes communs dans leurs expériences et à compléter les orientations existantes. Trois grandes questions de recherche ont été utilisées pour explorer l'incidence de la subjectivité des professionnels dans la sélection:

1. Quelles mesures figurent dans la pratique des professionnels lors du processus de sélection pour la préservation numérique?

2. Comment les professionnels perçoivent-ils l'influence de leurs rôles personnels (y compris l'expérience, les attitudes et les compétences) dans la création de l'objet intellectuel résultant d'un processus de sélection de préservation numérique?

3. Quels facteurs subjectifs récurrents sont identifiés dans le processus de choix des professionnels de la numérisation?

La recherche a ciblé des professionnels ayant des rôles et des fonctions dans les projets de numérisation. En discutant de l'information transmise par les archivistes et par les bibliothécaires inclus dans cette étude, nous avons utilisé la terminologie «participant». 
Sept participants de six établissements différents ont été choisis selon les méthodes suivantes. D'abord, les participants ont été sélectionnés à partir de leurs expériences probables dans des institutions locales, y compris des universités, des musées et des organismes culturels communautaires. Ensuite, une demande de participants a été envoyée à un forum Web (LinkedIn) pour les professionnels de la numérisation. Bien qu'il soit possible que les organismes soient différents, nous avons insisté pour que tous les participants aient travaillé sur des projets de numérisation afin de pouvoir parler de leurs expériences. Des résultats pertinents et significatifs sur les perceptions ou les croyances partagées étaient attendus en parlant avec un minimum de quatre à six professionnels. Enfin, nous nous sommes servis des données des cinq professionnels qui ont comblé nos attentes lors des entrevues et des processus d'enquête.

Nous avons utilisé une approche qualitative pour susciter des réponses sur la façon dont les participants ont expérimenté la sélection de la numérisation. Les entrevues étaient semi-structurées et accompagnées d'un questionnaire à choix multiples et de questions non dirigées. La nature subjective (c'est-à-dire expérientielle et abstraite) des données nécessitait une discussion interactive avec ces archivistes et bibliothécaires expérimentés. Ce processus d'entrevue semi-structurée a permis de couvrir des sujets spécifiques tout en reconnaissant que différents archivistes ou bibliothécaires opéraient selon différents champs d'application et politiques institutionnelles.

Trois entrevues ont été enregistrées lors de rencontres. La quatrième entrevue a été enregistrée lors d'une conversation téléphonique et la cinquième par courriel. Les entretiens audio ont été transcrits et un examinateur indépendant les a vérifiés. Les enquêtes de fond comprenaient sept questions et ont été menées après l'entrevue à l'aide d'un formulaire Web d'une page.

Une analyse des points communs dans les expériences des participants a permis de faire apparaître des thèmes récurrents qui couvraient ce que les participants considéraient dans leur processus de sélection. Ceux-ci ont été codés et utilisés dans notre évaluation. 


\subsection{Résultats}

\subsubsection{Contexte du participant}

Les cinq participants étaient âgés de 25 à 64 ans. Tous avaient de l'expérience dans la gestion de projets de numérisation. Quatre en avaient dans le catalogage ou la description, dans la préservation, dans la sélection des collections à numériser et avaient siégé à un comité consultatif. Parmi les autres expériences communément signalées figuraient le travail d'acquisition, la désignation des documents, des collections ou des fonds, l'évaluation des propositions d'inscription et la numérisation. Cette palette d'expériences suggérait une compréhension globale des projets de numérisation et non une focalisation sur un seul aspect de la numérisation. Le Tableau 1 donne un aperçu des antécédents des participants (marqués P1, P2, P3, P4 et P5).

\begin{tabular}{|c|c|c|c|c|c|}
\hline Participant & P1 & P2 & P3 & P4 & P5 \\
\hline Titre & $\begin{array}{c}\text { Directeur, } \\
\text { Services de } \\
\text { bibliothèque }\end{array}$ & $\begin{array}{c}\text { Bibliothécaire } \\
\text { en } \\
\text { numérisation, } \\
\text { Coordonnateur } \\
\text { de } \\
\text { numérisation } \\
\text { et livraison }\end{array}$ & $\begin{array}{c}\text { Directeur, } \\
\text { gestion des } \\
\text { documents et } \\
\text { archives }\end{array}$ & $\begin{array}{c}\text { Archiviste } \\
\text { universitaire } \\
\text { intérimaire }\end{array}$ & $\begin{array}{c}\text { Archiviste } \\
\text { aux Archives } \\
\text { générales de } \\
\text { l'État de [Pays] } \\
\text { - Archives } \\
\text { historiques de } \\
\text { [Région] }\end{array}$ \\
\hline $\begin{array}{l}\text { Fonction } \\
\text { actuelle }\end{array}$ & $\begin{array}{c}\text { Directeur, } \\
\text { bibliothèque }\end{array}$ & $\begin{array}{c}\text { Bibliothécaire } \\
\text { en } \\
\text { numérisation }\end{array}$ & $\begin{array}{l}\text { Archiviste, } \\
\text { directeur }\end{array}$ & $\begin{array}{c}\text { Analyste, } \\
\text { archiviste, } \\
\text { chef de projet }\end{array}$ & Archiviste \\
\hline $\begin{array}{l}\text { Nombre de } \\
\text { projets de } \\
\text { numérisation } \\
\text { dans son } \\
\text { expérience }\end{array}$ & 2 projets & 4 projets & 20 projets & 5 projets & 4 projets \\
\hline $\begin{array}{c}\text { Nombre } \\
\text { d'années } \\
\text { d'expérience } \\
\text { en sélection } \\
\text { pour } \\
\text { numérisation }\end{array}$ & $2-5$ ans & $\begin{array}{c}\text { Moins de } 2 \\
\text { ans }\end{array}$ & Plus de 15 ans & $2-5$ ans & $6-10$ ans \\
\hline
\end{tabular}

Tableau 1: Résumé des données sur les participants 
La quantité de projets, d'objets numériques créés ou d'années d'expérience n'a pas d'effet appréciable sur les attitudes, sur les observations des participants, sur la sélection ou le processus de numérisation en général. Les réponses des participants aux questions d'entrevue révèlent des perspectives venant d'une grande différence en termes de l'ampleur de leur travail. Néanmoins, cette différence ne distingue pas les opinions des participants.

\subsubsection{Quelles sont les étapes des processus de sélection pour numérisation adoptées par les professionnels dans leur pratique?}

Aucun participant n'avait mis en œuvre des politiques complètes et formelles pour leur processus telles que celles décrites dans la littérature. Les participants P2 et P4 ont travaillé selon une variété de procédures, qui ont été évaluées d'un projet à l'autre; tous deux envisageaient la mise en place d'un système formel. Même en l'absence de politiques institutionnelles formelles, les participants ont entrepris des démarches semblables à l'égard des projets (à l'exception de P5). Premièrement, on reconnaissait la nécessité de numériser des items ou des fonds particuliers. Ce besoin était généralement amorcé par une demande pour le matériel. Cette façon de procéder n'est pas celle que les participants préféreraient utiliser : ils aimeraient numériser en fonction de politiques institutionnelles (de manière continue et systématique) pour gérer leurs collections et leurs avoirs plutôt que de travailler de projet en projet. Dans certains cas, des donateurs ont contribué au financement de la numérisation. Ce qui a essentiellement déterminé la sélection (à l'exception de facteurs prohibitifs, par exemple les droits d'auteur).

Le modèle de sélection commun (similaire aux processus décrits dans la littérature) a commencé par une personne qui a proposé une partie de la collection pour la numérisation. Toutefois, les mises en candidature n'ont pas nécessairement été choisies selon une procédure prédéterminée de numérisation. La nomination pouvait provenir d'une demande extérieure à l'institution, d'archivistes ou de bibliothécaires responsables, ou encore d'un collègue désirant utiliser l'objet numérique résultant. Les nominations internes ont souvent été choisies parce que les matériaux devenaient inaccessibles en raison de leur dégradation physique ou de l'obsolescence du format. Dans certains cas, un processus de sélection a été lancé pour appuyer d'autres types d'activités que l'institution entreprenait ou 
pour répondre aux souhaits de la communauté d'utilisateurs désirant y accéder via le Web.

Dans le cas de P1, la sélection comprenait les collections et visait principalement à assurer un accès ininterrompu aux documents sujets à la dégradation ou aux formats obsolètes. Parce que le participant a procédé à l'identification de documents qui seraient en demande, il s'agissait en fait d'un projet basé sur la demande. P1 a pris des mesures spécifiques aux fins de son projet, mais pas sur la base d'une politique institutionnelle.

Après l'étape de la mise en candidature, les participants avaient généralement des discussions sur la nomination et les facteurs de sélection: c'était au fond une phase d'évaluation. Dans certains cas, la discussion avait lieu lors d'une réunion formelle entre pairs chargés d'examiner ce qui était proposé et de décider s'il fallait ou non poursuivre. Dans d'autres cas, la discussion était moins formalisée et faisait l'objet d'un examen collaboratif. Ces discussions servaient de vérification finale avant de soumettre leur sélection à la phase suivante du processus de numérisation. Si les discussions aboutissaient à une décision négative, la sélection n'était pas numérisée. En règle générale, les participants ont tenu compte des facteurs suivants:

- le contenu (par exemple: son importance, son exhaustivité, sa valeur éducative ou sa valeur promotionnelle);

- la personne qui génère la demande ;

- le résultat: est-ce que cela servirait réellement l'intention? ;

- la condition physique ;

- le format original ;

- les restrictions (par exemple: les accords sur les droits d'auteur ou les accords avec les donateurs de fonds);

- le caractère unique ou non.

En fin de compte, les étapes que les participants ont suivies dans la sélection pour la numérisation étaient similaires aux procédures recommandées dans beaucoup de guides existants dans la littérature, même s'ils ne se servaient pas de ces guides. 


\subsubsection{La perception des professionnels de leur influence personnelle dans la création de l'objet intellectuel résultant d'un processus de sélection pour la préservation numérique}

Les participants étaient conscients des connaissances personnelles sur lesquelles ils se sont appuyés ainsi que des objectifs de leur poste, de leur rôle et de leur employeur comme influences sur le processus de sélection. Une grande partie de ces connaissances provenaient de leur expérience, mais elles étaient également éclairées par les responsabilités de leur profession.

Les participants ont indiqué que la personnalisation des relations avec les utilisateurs les aidaient à comprendre de manière informelle leurs intérêts, le genre de documents numérisés qu'ils trouveraient utiles dans leurs institutions et quels autres types de contenu seraient appréciés. À titre d'exemple, P1 a déclaré: «On arrive à savoir ce que les gens enseignent et à connaître l'encadrement de leur recherche. Vous comprenez qu'ils forment la collectivité pour laquelle vous travailler sont votre peuple et vous les gardez à l'esprit tout le temps. » Ces relations et ces expériences ont influencé les approches des participants à la sélection, peut-être d'une façon indirecte. Les participants se sont critiqués à l'égard de cette influence, s'appuyant sur la discussion avec leurs collègues pour une perspective équilibrée dans la sélection. Par exemple, P3 a déclaré: «Je fais confiance à mon équipe, ils savent mieux que moi [...] Et mes intérêts personnels, j'essaie de les éliminer, de ne pas les mettre en premier, mais parfois je peux le faire sans m'en rendre compte. Mon équipe me le dira.»

Au-delà de connaître l'identité de l'utilisateur, les participants se sont servis de leur connaissance des attentes de ces utilisateurs comme une influence sur leurs jugements de sélection. Ils cherchaient à comprendre ce que les gens veulent obtenir en format numérique, en examinant, entre autres, les critères de recherche. En reconnaissant les attentes d'un utilisateur en matière d'accès, les participants peuvent aborder la sélection de certains documents en tenant compte des besoins des chercheurs une fois les documents numérisés.

Les participants ont noté que les utilisateurs veulent avoir un accès électronique à tout: selon les commentaires des utilisateurs, et selon l'observation des tendances actuelles, si quelque chose n'est pas accessible 
par le biais de la navigation et des recherches en ligne, ils deviennent confus et croient que l'objet recherché n'existe pas. Les utilisateurs ne pensant pas aller visiter physiquement des archives ou une bibliothèque pour trouver ce qu'ils recherchent, les participants ont jugé nécessaire de mieux leur faire connaître leurs fonds ou leurs collections pour les attirer. Ainsi, dans le cadre de leurs responsabilités professionnelles, les participants se sont impliqués dans des activités telles que: la production d'expositions en ligne; la création de sites Web permettant des recherches par motclé de documents numérisés; la création de versions numériques des instruments de recherche existants; et la création d'images miniatures pour la navigation et pour la prévisualisation.

Une attitude dominante parmi les participants était que tout devait être numérisé, mais les participants ont reconnu qu'ils n'avaient pas la capacité de satisfaire toutes les attentes des utilisateurs. Ils ont puisé dans leurs expériences en sélectionnant des items qui attireraient les utilisateurs aux matériaux numérisés. À titre d'exemple, ils ont reconnu que l'augmentation du trafic Web dépend de la sensibilisation afin que les utilisateurs sachent que les objets numérisés sont disponibles. P3 a noté que le fait de mesurer quels médias sociaux recueillent plus de commentaires a appuyé sa décision de numériser davantage de photos. De bons taux de réponse à partir du service de partage de photos Flickr pourraient influer sur son jugement sur le choix en priorité des images pour la numérisation.

Une familiarité intellectuelle avec le contenu de leurs avoirs a influencé la plupart des jugements de sélection des participants; cette familiarité s'est développée au fil du temps. La question se manifeste de différentes façons. D'abord, elle est fortement liée aux relations avec les utilisateurs, car il est crucial de savoir quel contenu satisfera les besoins des utilisateurs pour être en mesure de bien les servir. La familiarité avec le contenu a également soutenu la prise de décision qui devait faire face à l'actualité. À titre d'exemple, P4 faisait mention de sa familiarité avec certains documents liés à la Première Guerre mondiale, en appui aux chercheurs qui s'y intéressaient dû au centenaire de la guerre. P3 a mentionné le prochain anniversaire de son institution, qui a donné lieu à une demande de documents témoignant de son passé pour mieux le faire connaître. Ces requêtes démontrent une connaissance des sujets que les participants savaient être en demande. Les participants ont équilibré la valeur de faire valoir ce contenu par rapport à l'exposition d'autres contenus d'intérêt 
plus général. Cela était possible en raison de la familiarité qu'eux et leurs collègues ont de leurs avoirs.

Les participants ont également expliqué comment la connaissance des objectifs de leurs institutions pouvait influencer leur sélection. En fonction de la nature des documents, les participants ont jugé que quelques-uns étaient plus conformes aux objectifs institutionnels. À l'inverse, comme l'expliquait P3, elle pourrait, sur la base de son expérience et de sa compréhension de I'histoire de l'institution, juger certains contenus potentiellement préjudiciables à la réputation de l'institution ou pénibles pour des personnes vivantes. Elle préservait consciencieusement ces documents, mais elle leur assignait une priorité inférieure pour la numérisation et pour la dissémination. De son côté, P2 a souligné le but de répondre aux besoins de la recherche de l'intérieur de l'institution ou d'ailleurs. Afin de combler les objectifs institutionnels, les participants devaient conceptualiser et prendre à cœur ce que les documents qu'ils ont choisi de numériser soutiendraient.

Dans leurs rôles professionnels, les participants ont utilisé leurs connaissances pour appuyer des décisions portant sur des événements dans la sphère publique ou sur des questions d'actualité. P4 a parlé d'un exemple des fonds d'archives d'une autre institution, qui comprenaient des documents risquant de causer des dommages s'ils étaient diffusés en ligne, à un public potentiellement large. Elle a dit: «la décision ultime était que cette information ne soit pas divulguée. [...] Je m'y suis conformée parce que je pense qu'elle peut nuire à l'environnement ou à une certaine situation et donc [...] éthiquement et moralement je pense que je pourrais le faire. » Ainsi, ce qu'un professionnel juge approprié pour la numérisation (et donc pour un accès accru) peut avoir un impact sur la sphère publique.

En résumé, les participants ont identifié comment la sélection pouvait être influencée en raison des connaissances acquises lors du travail par leurs relations avec les utilisateurs ou les collègues. Le sentiment que tout devrait être numérisé, résultant de la connaissance des attentes de recherche et d'accès des utilisateurs, a influencé leur sélection. Cette influence peut être attribuée à une compréhension acquise par le biais du rôle professionnel de l'archiviste ou du bibliothécaire. Connaître le contenu de leurs fonds ou collections a aidé les participants à atteindre les objectifs institutionnels, ce qui a aussi influencé la sélection. Enfin, 
les participants ont tenu compte de leurs rôles professionnels lorsqu'ils considéraient les préoccupations éthiques concernant ce que la disponibilité d'un objet numérisé signifierait pour la sphère publique.

\subsubsection{Quels sont les facteurs subjectifs récurrents dans les processus qu'entreprennent les professionnels dans leurs choix pour la préservation numérique?}

Les phases principales du processus de sélection, telles que décrites par les participants, peuvent être regroupées et résumées sous forme de nomination, de discussion des nominations (évaluation) et de la décision de numériser. La délimitation de ces phases a été influencée par certains facteurs récurrents et subjectifs liés aux connaissances personnelles et aux perspectives professionnelles des participants. Ces facteurs récurrents de la subjectivité de sélection comprenaient:

1. les relations avec les utilisateurs et les collègues;

2. la sensibilisation aux attentes et aux besoins des utilisateurs;

3. la compréhension intellectuelle du contenu;

4. la compréhension des objectifs institutionnels;

5. la position sur la mesure dans laquelle son rôle doit être actif dans la sphère publique ;

6. I'identification de la valeur.

Les cinq premiers facteurs ressortent des réponses des participants à la deuxième question de l'entrevue, sur l'influence des rôles personnels. Le sixième facteur, l'identification de la valeur, a été exposé dans d'autres commentaires. À titre d'exemple, les participants abordaient le sujet de la valeur d'un point de vue intellectuel, ce qui présente un problème pour les chercheurs. Les participants ont parlé de la valeur de leurs fonds d'archives en termes de leurs points forts. P1 s'est servie de sa connaissance selon laquelle sa communauté reconnaissait un congrès en particulier comme un moment clé pour les arts. Elle a également compris que quelques poèmes de sa collection avaient acquis une certaine renommée dans sa communauté. Les participants identifiaient intuitivement ce qui constitue un point saillant, basé sur leur expérience professionnelle ou leur conscience professionnelle. 
Une autre façon de déterminer la valeur était fondée sur la connaissance de l'exhaustivité. P4 s'est appuyée sur ses connaissances professionnelles de la complétude d'un fonds d'archives dans son institution. Si elle savait que des portions significatives existaient dans d'autres institutions ou manquaient dans ses avoirs, elle était susceptible de juger que ça ne valait pas la peine de numériser sa portion des documents. Elle a déclaré:

Une fois que nous avons choisi un fonds, ensuite nous nous assurons [...] qu'il est assez complet pour produire une véritable histoire. Je trouve parfois qu'on ne reçoit qu'un ou deux items du donneur, ou bien il pourrait être une donation isolée, donc vous ne voulez pas nécessairement les numériser.

Elle voulait offrir accès à une histoire complète pour les utilisateurs et si elle comprenait qu'elle ne pouvait fournir qu'un fragment, elle jugeait que c'était moins important de le numériser.

La valeur probante à l'institution concernait les participants P3 et P4 ; tous deux avaient également des responsabilités en matière de gestion des documents. P3 a parlé de la valeur probante des documents au service de son université et de la valeur informationnelle. À titre d'explication, P3 a offert un exemple hypothétique: s'il y avait des documents d'archives qui témoignaient d'un spectacle offert par les Beatles dans une des installations de l'établissement, ceux-ci auraient une grande valeur informationnelle en raison de leur importance pour l'ensemble de la communauté, mais ils auraient peu d'impact sur ses objectifs. Cette reconnaissance exigeait une prise de conscience personnelle de la signification culturelle et historique d'un tel événement et de sa relation dans le contexte de sa fonction. Les participants ont également considéré la valeur en termes de matériaux qui pourraient attirer plus d'utilisateurs à leurs institutions. Des documents qui pourraient permettre la collaboration avec d'autres institutions aux fins des expositions qui attireraient l'attention pourraient être considérés comme plus importants à numériser.

Les guides de numérisation conseillent de considérer des facteurs fréquemment récurrents tels que la fragilité ou la fréquence d'utilisation pour sélectionner des matériaux. Comparer ces types de facteurs objectifs aux facteurs identifiés dans cette étude, distingue ce qui résulte de la subjectivité. Plutôt que d'être fondés sur des caractéristiques physiques 
ou statistiques objectivement identifiables, les facteurs de cette étude découlent des connaissances ou des perspectives que les participants eux-mêmes ont exposées.

\section{DISCUSSION ET RECOMMANDATIONS}

Les jugements subjectifs que font les archivistes ou les bibliothécaires figurent dans le choix des matériaux pour la numérisation. Nous devrions nous préoccuper de ces jugements - qui reposent en grande partie sur les connaissances personnelles et les perspectives professionnelles parce que la subjectivité d'un individu influe sur la sélection d'une manière qui n'est pas transparente pour ceux qui utiliseront les objets numérisés, ni pour ceux qui maintiendront les documents numérisés dans l'avenir.

Est-ce que I'on pourrait ajouter aux étapes habituelles du processus de sélection pour numérisation des procédures uniformes pour traiter la subjectivité? En raison de la nature idiosyncrasique de la subjectivité, il peut ne pas être possible de faire un guide complet sur la façon d'aborder chaque facteur subjectif dans une phase de sélection. En effet, la littérature, reconnaissant que la subjectivité existe comme un aspect des décisions de sélection, n'offre pas de procédures pour en tenir compte comme cela se fait pour les facteurs objectifs. En outre, il se peut que le développement d'une procédure uniforme puisse ne pas être la meilleure façon de traiter la subjectivité.

La reconnaissance de I'influence subjective dans la sélection et l'enregistrement formel des jugements subjectifs, comme un ensemble de notes de sélection associés à l'objet numérique, pourrait se révéler une technique utile. II permettrait une plus grande transparence sur la manière dont les facteurs de subjectivité sont sélectionnés. Une telle note permettrait aux utilisateurs d'interpréter et de comprendre le contexte de ce à quoi ils ont accès, et cela pourrait être fait sans exiger beaucoup de détails. Nous pouvons comprendre comment cela pourrait fonctionner en considérant d'abord, pourquoi nous numérisons (en regardant la pratique), ensuite, le produit de la numérisation et, enfin, I'influence du rôle professionnel (attentes et responsabilités à l'égard de l'objet numérisé, comme identifiées par les facteurs récurrents de subjectivité). 


\subsection{Pourquoi numérisons-nous?}

Lorsqu'elle est motivée par le besoin de préservation, la numérisation se fait principalement pour permettre l'accès. Les participants à cette étude ont exprimé des points de vue qui étaient cohérents avec la littérature sur les objectifs d'accès et de conservation: la numérisation satisfait aux exigences essentielles d'accès des utilisateurs, car elle compense I'usure des matériaux originaux trop fragiles à manipuler. En raison des contraintes liées au financement, au personnel, à l'équipement, au temps requis pour la numérisation et à la mise en place d'entretien continu supplémentaire, on ne peut pas tout numériser. Ce qui conduit à la sélection qui repose sur les facteurs et les jugements documentés dans la littérature et dans cette étude. Les archivistes et les bibliothécaires doivent développer des processus de sélection pour la numérisation afin de répondre aux besoins d'accès des utilisateurs.

\subsection{Le produit de la numérisation}

L'acte de numériser crée un objet numérique qui n'existait pas auparavant. La responsabilité professionnelle de donner accès à ce nouvel objet nécessite la compréhension du processus de sélection. Ce nouvel objet, ce substitut numérique de l'objet analogique original, a des caractéristiques différentes de l'analogique. La description de l'objet numérisé donne une idée de ce qui est attendu du rôle et de la responsabilité du professionnel.

Conway offre une description utile de l'objet numérisé ; il explique que nous n'essayons pas de préserver l'intégrité physique d'un objet, mais «de spécifier la création et le maintien de l'objet dont l'intégrité intellectuelle est sa principale caractéristique» (Conway, 2010). L'objet numérisé ne traduit pas les caractéristiques physiques de l'original, ni sa proximité à d'autres objets dans une collection, ni le contexte physique. II est son propre objet avec ses propres propriétés, tout en conservant un lien intellectuel à sa source physique.

Les archivistes font la distinction entre le numérique et sa source analogique (Jones et Beagrie, 2001), et ils nient souvent que l'objet numérisé peut servir de remplacement pur. Compte tenu des ressources et des efforts nécessaires pour préserver les objets numériques, conjugués à la situation budgétaire souvent difficile des institutions de la mémoire, 
cette distinction revêt une importance considérable. Ceux qui créent et entretiennent de nouveaux objets numériques offrent l'accès à un document auquel les utilisateurs $n$ 'avaient pas accès auparavant ou auquel ils peuvent désormais accéder de façon nouvelle.

\subsection{Le rôle professionnel (attentes et responsabilités)}

Il peut être utile de considérer comment l'évaluation exige un jugement professionnel spécialisé. Notant l'importance de la perspective du professionnel au cours de l'évaluation, Verne Harris écrit:

Les valeurs de l'évaluateur, la qualité de son travail, ses perspectives, son interaction avec l'agence créatrice des documents, son engagement avec la politique qu'il met œuvre, etc. influencent l'évaluation. L'évaluateur n'identifie pas simplement les documents comme ayant une valeur archivistique; il ou elle est plutôt le créateur de valeur archivistique. [...] Donc, le dossier d'archivage fournit une fenêtre dans le processus d'évaluation autant que dans toute autre chose. Cela nécessite une approche qui considère le rapport ou l'étude d'évaluation comme un record, un texte avec un statut ontologique très spécifique. (Harris, 2002)

Dans le contexte de la numérisation, I'archiviste ou le bibliothécaire crée quelque chose qui, une fois accédé, apportera une quantité implicite d'information sur les processus et les jugements qui ont abouti à sa création: c'est-à-dire des informations sur la subjectivité de l'individu. Dans notre étude, P4 a expliqué comment un haut fonctionnaire de son institution pouvait demander des documents afférant à un important événement à venir, en s'appuyant sur le jugement de l'archiviste pour déterminer ce qui serait pertinent et utile. Les facteurs subjectifs auxquels P4 aurait eu recours dans son jugement proviennent de sa propre expérience, contrairement à des facteurs de sélection objectifs qui pourraient autrement étayer une décision impartiale. Les actions des participants dans cette étude ont été influencées en partie à partir de leur expérience professionnelle et de leur connaissance de la sphère publique. Cette sensibilisation a été étendue à l'évaluation des avantages ou des inconvénients potentiels pouvant résulter de la numérisation des 
documents. Les objets créés dans un processus de numérisation forment une fenêtre sur la sélection, tout comme le dossier archivistique de Harris ouvre sur l'évaluation.

\subsection{Aborder l'impact de la subjectivité}

L'enregistrement des notes portant sur la sélection de numérisation serait semblable aux notes de portée et contenu, qui fournissent aux utilisateurs ayant accès aux documents d'archives des informations et un contexte pour ce qu'ils trouvent. Dans le cas de l'objet numérisé, le contexte de son existence devient plus complet avec des informations sur la raison pour laquelle cet objet en particulier a été numérisé. Les notes de sélection de numérisation pourraient permettre à un professionnel de révéler ce qui est pris en compte dans ses jugements. Bien que la tâche ajouterait de l'effort, de telles notes rendraient transparente l'influence de la subjectivité afin que d'autres puissent prendre en considération les facteurs de production du document qu'ils utilisent ou maintiennent.

Une telle documentation sur la sélection pourrait commencer par prendre connaissance de l'information ou de la justification des facteurs récurrents liés aux connaissances et aux perspectives personnelles des archivistes ou des bibliothécaires. À titre d'exemple, il peut être suffisant $d^{\prime}$ inclure une note intitulée "Conscience des attentes et des besoins des utilisateurs», qui pourrait déclarer quelque chose à l'effet de "Connu pour avoir été utilisé dans une classe sur le surréalisme». Une telle note informerait sur l'existence de l'objet sélectionné. Examinons quelquesunes des raisons pour lesquelles les connaissances et les perspectives personnelles d'un archiviste ou d'un bibliothécaire pourraient contribuer à la compréhension par les utilisateurs du contexte de ce qui est évalué.

\subsection{Connaissances personnelles}

Sélectionner quelque chose à numériser nécessite de penser aux hypothèses des utilisateurs envers I'accès à l'objet numérisé. La connaissance des attentes des utilisateurs, du contenu et des objectifs institutionnels que les professionnels acquièrent à travers l'expérience est primordiale. L'utilisation de ces connaissances était évidente dans les réponses des participants sur le thème des attentes des utilisateurs; ils ont pensé aux utilisateurs qui ont accès aux documents, mais leurs 
réflexions ne sont pas transparentes pour les utilisateurs. P2 a déclaré qu'un utilisateur

était tellement agacé par le fait que nous avons continué à faire cette numérisation où nous ne montrons qu'une partie. [...] Pour lui, ce qu'il voulait dire, c'est qu'il trouverait cinq lettres, mais il n'aurait aucune idée si c'était toutes les lettres, si c'était toutes les lettres qui ont été numérisées, ou si ce n'était que les lettres qui avaient été sélectionnées.

Nous devons essayer de comprendre les hypothèses que les utilisateurs feront en percevant ce qu'ils trouvent, mais nous ne pouvons pas anticiper toutes les façons possibles qu'un utilisateur peut percevoir les documents auxquels il accède. Les objets numérisés peuvent être accessibles à partir d'une variété d'emplacements et de dispositifs, qui ne transmettent pas et sont sans rapport avec le contexte de toute l'étendue physique des sources analogiques. Les objets numériques peuvent être incorporés de façon inattendue, en devenant accessibles à partir d'autres objets qui $n^{\prime}$ ont rien à voir avec l'original. Des exemples populaires de ceci existent dans l'échantillonnage de musique ou des œuvres d'art. Si les utilisateurs connaissaient la manière dont le rôle du professionnel a influencé la sélection, ils pourraient interpréter de manière plus critique ce à quoi ils ont accès. Hypothétiquement, dans l'exemple des lettres numérisées par le participant P2, I'utilisateur pourrait ne pas s'inquiéter parce qu'au-delà de connaître l'étendue de ce qui a été numérisé, il pourrait comprendre comment seulement quelques lettres ont été choisies pour la numérisation.

\subsection{Perspectives professionnelles}

En proposant des moyens de juger de la valeur intellectuelle, des ouvrages tels que ceux de Hazen confrontent les professionnels avec le problème de la valeur (Hazen et al., 1998). Parmi les participants à cette étude, la valeur (le contenu et l'importance de l'information) semblait être jugée selon les points déterminants de l'établissement (ce qui exige la connaissance des objectifs de l'établissement), les articles tenus en estime par l'utilisateur ou la communauté d'utilisateurs potentiels (nécessitant la connaissance des utilisateurs) et les idées telles que l'exhaustivité des matériaux pour la numérisation (nécessitant la connaissance du 
contenu). Ces domaines n'adhèrent pas à des facteurs objectifs bien définis; par contre, ils impliquent des perspectives professionnelles. Les utilisateurs évaluent le contenu numérisé pour leurs propres raisons, qui ne correspondent pas nécessairement à celles en jeu dans la sélection pour la numérisation. Les jugements de valeur des archivistes et des bibliothécaires nécessitent des données objectives, mais, en raison du caractère intellectuel et expérientiel, ces jugements impliquent également des zones grises. Communiquer les jugements de valeur qui ont été sélectionnés aiderait à fournir un contexte pour ce qui est accessible numériquement.

Les contenus marquants constituent un critère de valeur difficile à cause de la durée d'utilisation de ces contenus. L'exemple de P1 montre sa capacité à juger ce que les communautés intéressées à certains éléments considèrent comme important. Sa connaissance provient principalement des interactions régulières avec les professeurs qui ont utilisé les items. Sa communauté a une compréhension actuelle de la valeur des matériaux, mais I'institution maintiendra ces matériaux pendant longtemps; la valeur peut évoluer. Hazen et ses collègues proposent des questions d'évaluation pour déterminer si la valeur dans le temps justifie l'accès rendu disponible par un projet de numérisation (Hazen et al., 1998). Le nombre d'idées-clés pour juger de la valeur intellectuelle pourrait augmenter en proportion des diverses caractéristiques numériques, des documents d'éminents spécialistes, des agrégations et des collections interchangeables plus révélatrices du contexte que les articles seuls et des modèles d'utilisation. Identifier la valeur intellectuelle de cette façon sensibilise au cours d'un processus de sélection, mais dépend également des perspectives des archivistes et des bibliothécaires. Présupposer ce qui sera utile à un chercheur est une tâche difficile; la valeur des matériaux varie de façon imprévue et selon les objectifs du chercheur. Lorsqu'on juge la valeur en fonction des faits saillants de la collectivité ou des critères intellectuels, la perspective du professionnel pourrait être consignée. Cette transparence permettrait aux utilisateurs de valider selon leurs interprétations de ce à quoi ils ont accès.

Nous devons également envisager une activité professionnelle dans la sphère publique. Une lentille sur l'interaction avec la sphère publique vient de la pratique de la numérisation des matériaux pour établir un lien avec d'autres collections. Les institutions actives dans la présentation de leurs contenus numérisés s'impliquent dans la vie publique; leurs choix reflètent certains jugements subjectifs. Un exemple est le site Web d'un 
radiodiffuseur de nouvelles qui relie des clips de télévision et de radio de ses archives pour fournir le contexte historique sur des sujets courants. Numériser des objets pour les lier à certains sujets par opposition à d'autres est un jugement sur le type de recherche ou d'intérêt qui sera associé avec eux. Cela met les archivistes et les bibliothécaires dans un rôle où ils créent quelque chose prévu pour un sujet plutôt que de permettre l'utilisation d'une perspective impartiale.

Enfin, nous devrions réfléchir sur la façon dont les participants se sont appuyés sur les connaissances qu'ils ont acquises grâce à leurs relations interpersonnelles. Dans les établissements d'enseignement, comme P1 I'expliquait, il était important de garder à l'esprit sa connaissance du genre de matériel que les enseignants aimeraient utiliser pour leurs classes. P2 a constaté être plus disposé à entreprendre des projets de numérisation lorsque le projet comprenait des utilisateurs qui avaient participé à des projets précédents. Une note indiquant que quelque chose avait été numérisé au cours d'un travail répété avec des intervenants internes pourrait aider les utilisateurs futurs à comprendre que leur accès pourrait avoir été le résultat d'une exigence d'accès institutionnel.

\section{CONCLUSION}

Cette étude a commencé par examiner l'impact de la subjectivité sur les processus de sélection pour la numérisation et visait à compléter les orientations existantes. Tout en n'adhérant pas à un ensemble spécifique de directives de numérisation, les participants ont pratiqué des étapes similaires durant la phase de sélection: nomination (en utilisant principalement des facteurs objectifs), discussion ou évaluation de candidatures et décision de numériser (ou non) le matériel proposé. Les participants ont indiqué que leurs expériences, leurs attitudes et leurs compétences influençaient leurs jugements de sélection. Celles-ci allaient de leur connaissance personnelle des usagers, des attentes des utilisateurs et du contenu de leurs avoirs, aux obligations vis-à-vis des objectifs de leur institution et des perspectives professionnelles sur la nature de leur activité dans la sphère publique.

Les expériences des participants suggèrent, comme dans la littérature, que les sélections peuvent façonner la compréhension et le discours d'un sujet. II peut ne pas être possible de sélectionner uniquement en fonction 
de facteurs objectifs, mais la documentation des facteurs qui ont influencé la sélection pourrait aider les utilisateurs à percevoir et à comprendre ce à quoi ils ont accès dans un contexte numérique. Une telle pratique expliquerait comment les archivistes et les bibliothécaires s'appuient sur leurs connaissances personnelles et leurs perspectives professionnelles.

Une des limites de cette étude est que la plupart des participants ont travaillé dans le cadre d'une institution universitaire; I'un d'eux travaillait dans un contexte gouvernemental. Cela peut réduire l'éventail des perspectives sur les processus organisationnels et sur les pairs. Une version à grande échelle de cette étude examinerait les perspectives d'une variété d'organismes, améliorant notre compréhension des expériences de numérisation des archivistes et des bibliothécaires.

Une étude plus approfondie, qui se concentrerait sur les impressions des utilisateurs concernant la façon dont ils perçoivent le contexte de ce qui est disponible pour eux lors de l'accès aux objets numérisés, serait précieuse. Elle permettrait de développer davantage un moyen de divulguer les informations nécessaires sur les objets sélectionnés dans les fonds d'archives et les collections de bibliothèques. II serait utile de rechercher et de tester différentes méthodes de divulgation de l'information de sélection subjective afin de déterminer ce qui serait le plus efficace pour les utilisateurs.

Joshua Chalifour et Eun G. Park

\section{BIBLIOGRAPHIE}

ANDERSON, C. (2006). Ethical Decision Making for Libraries. Oxford, Royaume-Unis: Chandos Publishing Ltd.

ASSOCIATION FOR LIBRARY COLLECTIONS \& TECHNICAL SERVICES, PRESERVATION AND REFORMATTING SECTION. (Juin 2007). Definitions of Digital Preservation. Washington, D.C. : Conférence de la American Library Association.

BAILLARGEON, S. (2014). La mémoire de I'art. Le Devoir, 8 octobre, édition finale. 
BÜLOW, A., AHMON, J. et SPENCER, R. (2011). Preparing Collections for Digitization. Londres, Royaume-Unis: Facet Publishing, en association avec les National Archives.

COLE, R. et HACKETT, C. (2010). Search vs. Research: Full-text Repositories, Granularity, and the Concept of «Source» in the Digital Environment. Dans C. Avery et M. Holmlund Better off Forgetting?: Essays on Archives, Public Policy, and Collective Memory. Toronto, Ontario: University of Toronto Press.

CONSEIL CANADIEN DES ARCHIVES. (2002). Digitization and Archives. Repéré le 3 avril 2013, à http://www.cdncouncilarchives.ca/digitization en.pdf.

CONSEIL CANADIEN DES ARCHIVES. (2002). Numérisation et archives. Repéré le 25 novembre 2016, à http://www.cdncouncilarchives.ca/ digitization fr.pdf.

CONWAY, P. (2010). Preservation in the Age of Google: Digitization, Digital Preservation, and Dilemmas. Library Quarterly 80(1), 61-79.

COOK, T. (2001). Archival Science and Postmodernism: New Formulations for Old Concepts. Archival Science. 1(1), 3-24.

FRESKO, M. et TOMBS, K. (1998). Digital Preservation Guidelines: the State of the Art in Libraries, Museums and Archives. Luxembourg: European Commission, Directorate General XIII/E4. Repéré le 28 mai 2014, à ftp://ftp.cordis.lu/pub/ist/docs/digicult/study1.doc.

GERTZ, J. (2000). Selection for Preservation in the Digital Age. Library Resources \& Technical Services 44(2), 97-109.

HAMPSHIRE, E. et JOHNSON, V. (2009). The Digital World and the Future of Historical Research 1. Twentieth Century British History. 20(3), 396-414.

HARRIS, V. (2002). The Archival Sliver: Power, Memory, and Archives in South Africa. Archival Science. 2(1-2), 63-86.

HAZEN, D., HORREL, J. et MERRILL-OLDHAM, J. (1998). Selecting Research Collections for Digitization. Washington, D. C. : Council on Library and Information. Repéré le 14 septembre 2014, à http://www.clir.org/pubs/ reports/hazen/pub74.html/pub74.html\#intellectual. 
HUGHES, L. M. (2004). Digitizing Collections: Strategic Issues for the Information Manager. Londres, Royaume-Unis: Facet.

JENKINSON, H. (1922). A Manual of Archive Administration Including the Problems of War Archives and Archive Making. Oxford, Royaume-Unis: The Clarendon Press.

JONES, M. et BEAGRIE, N. (2001). Preservation Management of Digital Materials: a Handbook. Londres, Royaume-Unis: The British Library for Resource, the Council for Museums, Archives and Libraries.

MCELRATH, D. P. (2005). Why We Collect: Curators, Collectors, and the Urge to Acquire. Dans Y. Carignan Who Wants Yesterday's Papers?: Essays on the Research Value of Printed Materials in the Digital Age. Maryland: Scarecrow Press.

MCILWAINE, J., COMMENT, J.-M., DE WOLF, C., PETERS, D., JUSTRELL, B., VARLAMOFF, M.-T. et KOOPMAN, S. (2002). Guidelines for Digitization Projects for Collections and Holdings in the Public Domain, Particularly those Held by Libraries and Archives. https://www.ifla.org/ files/assets/preservation-and-conservation/publications/digitizationprojects-guidelines.pdf

MOSS, M. (2005). The NINCH Guide to Good Practice in the Digital Representation and Management of Cultural Heritage Materials: Technical Guidelines for Digital Cultural Content Creation Programs. American Archivist. 68(1), 40.

RÉSEAU CANADIEN D'INFORMATION SUR LE PATRIMOINE. (2000). Guide à l'intention des gestionnaires chargés de la planification et de la mise en oeuvre de projets d'informatisation. Repéré le 25 novembre 2016. http://canada.pch.gc.ca/fra/1447160408221/1447164205893

ROOSA, M. (2005). Some Thoughts on the Race Against Time and Inherent Vice: Library Preservation in the Late Twentieth Century. Dans Y. Carignan, Who Wants Yesterday's Papers?: Essays on the Research Value of Printed Materials in the Digital Age. Maryland: Scarecrow Press.

SCHWARTZ, J. M. et COOK, T. 2002. Archives, Records, and Power: the Making of Modern Memory. Archival Science. 2(1-2), 1-19. 
SITTS, M. K. (2000). Handbook for Digital Projects: a Management Tool for Preservation and Access. Andover, MA: Northeast Document Conservation Center.

SMITH, A. (1999). Why Digitize? Council on Library and Information Resources. Repéré le 29 novembre 2016, http://www.clir.org/pubs/ reports/pub80-smith/pub80.html\#access

STERNFELD, J. (2011). Archival Theory and Digital Historiography: Selection, Search, and Metadata as Archival Processes for Assessing Historical Contextualization. American Archivist, 74(2), 544-575.

STILL IMAGE WORKING GROUP. (2009). Digitization Activities Project Planning and Management Outline. U.S. National Archives and Records Administration. Repéré le 13 mai 2014, http://www.digitizationguidelines. gov/guidelines/DigActivities-FADGl-v1-20091104.pdf

UNITED KINGDOM. PARLIAMENTARY ARCHIVES. (2008). Digitisation Guidelines. Repéré le 28 mai 2014, à http://www.parliament.uk/ documents/upload/digitisation-guidelines.pdf

UNITED KINGDOM. THE NATIONAL ARCHIVES. (2009). Corporate Preservation Policy. [Richmond] : 3-4. Repéré le 28 mai 2014, à https:// web.archive.org/web/20111027080106/http://www.nationalarchives. gov.uk/documents/tna-corporate-preservation-policy-2009-websiteversion.pdf

VOGT-O'CONNOR, D. (2000). Selection of Materials for Scanning. Dans Maxine K. Sitts Handbook for Digital Projects: a Management Tool for Preservation and Access. Massachusetts: Northeast Document Conservation Center.

ZHANG, A. B., et GOURLEY, D. (2008). Creating Digital Collections: a Practical Guide. Oxford, Royaume Unis: Chandos Pub. 